\title{
SYNTHESIS OF HUMAN-BIKE KINEMATIC STRUCTURES FOR DIRECT KINEMATIC ANALYSIS
}

\author{
A. Handke*, J. Bałchanowski ${ }^{* *}$
}

\begin{abstract}
The work concerns the analysis of possible kinematic structures of a human lower limbs set in combination with a bicycle. The selection of the appropriate kinematic structure was carried out in a manner allowing to perform numerical studies on the created man-bicycle model. The research was aimed at the elimination of passive constraints present in common models intended for kinematics and dynamics studies of the drive transfer from human lower limbs to the bicycle crank. Selected models have been used to obtain information on the driving moments in the hip and knee joints, as well as the loads from the reaction forces. Selected parameters were obtained by inverse kinematics based on displacement characteristics in individual joints. The research was carried out in an environment designed for dynamic simulation studies of multibody systems.
\end{abstract}

Keywords: bicycle, biomechanics, kinematic structure, inverse kinematics

\section{Introduction}

The human motion organ is characterized by a complex kinematic system with many degrees of freedom. This system is characterized by spatial movable relations between particular bones of the lower and upper limbs. In the case of the lower limb, the most common number is the 7 degrees of freedom between the pelvis and the foot of each limb (Olinski, 2016). This means that the lower limb forms a redundant system thanks to which the mobility of the entire limb is increased. Analyzing the existing types of foot-powered bicycle construction, it can be noticed that their properties are dictated mainly by driving considerations (Pucher, 2011). Many scientific papers have been devoted to the analysis of the human ability to transfer the drive from the gait apparatus to the chassis of the bicycle. Therefore, this work is the introduction to a broader biomechanical analysis of the human-bicycle system.

While walking, a man uses the fully spatial character of movements performed by lower limbs. This creates an additional challenge during the transition to a planar coordinate system in which the kinematic scheme of the bicycle is described most often. By entering a simplified model of the human lower limb into a planar coordinate system, it is possible to freely connect the human movement apparatus to transfer the drive directly to the driving part, which is the bicycle crank. Taking into account the mobility of a single human lower limb in a planar system and the use of both limbs while riding a bicycle, the conditions of human restraint to the bicycle frame should be selected. The problem concerns the adjustment of the human kinematic scheme together with the kinematic scheme of the bicycle, which in its drive system has only one degree of freedom. A man riding a bike usually takes two positions: sitting and standing (Arkesteijn, 2016). This work focuses mainly on pedaling in a sitting position omitting the upper limb participation in the adjustment of the driving position.

Assis. Prof. Artur Handke, PhD.: Department of Biomedical Engineering, Mechatronics and Theory of Mechanisms, Wroclaw University of Science and Technology, Łukasiewicza Street 7/9; 50-010, Wrocław; PL, artur.handke@pwr.edu.pl

Assoc. Prof. Jacek Bałchnowski, PhD., DSc.: Department of Biomedical Engineering, Mechatronics and Theory of Mechanisms, Wroclaw University of Science and Technology, Łukasiewicza Street 7/9; 50-010, Wrocław; PL, jacek.balchanowski@pwr.edu.pl 


\section{Selection of the structure and kinematic scheme of the human-bicycle system}

In the case of the models used to simulate the inverse dynamics task, most often the pelvis fixation relative to the bicycle frame is taken into account (Fig. 1a) as well as fixing the foot to the pedal. The kinematic scheme established in the planar system is characterized by mobility equal to 1 and is insufficient in combination with 6 degrees of freedom of both limbs, derived from 6 active joints: 2 hips, 2 knees and 2 ankles. Such model only allows to perform the analysis of the inverse kinematics task, however, it does not allow to determine the active moments of individual joints.
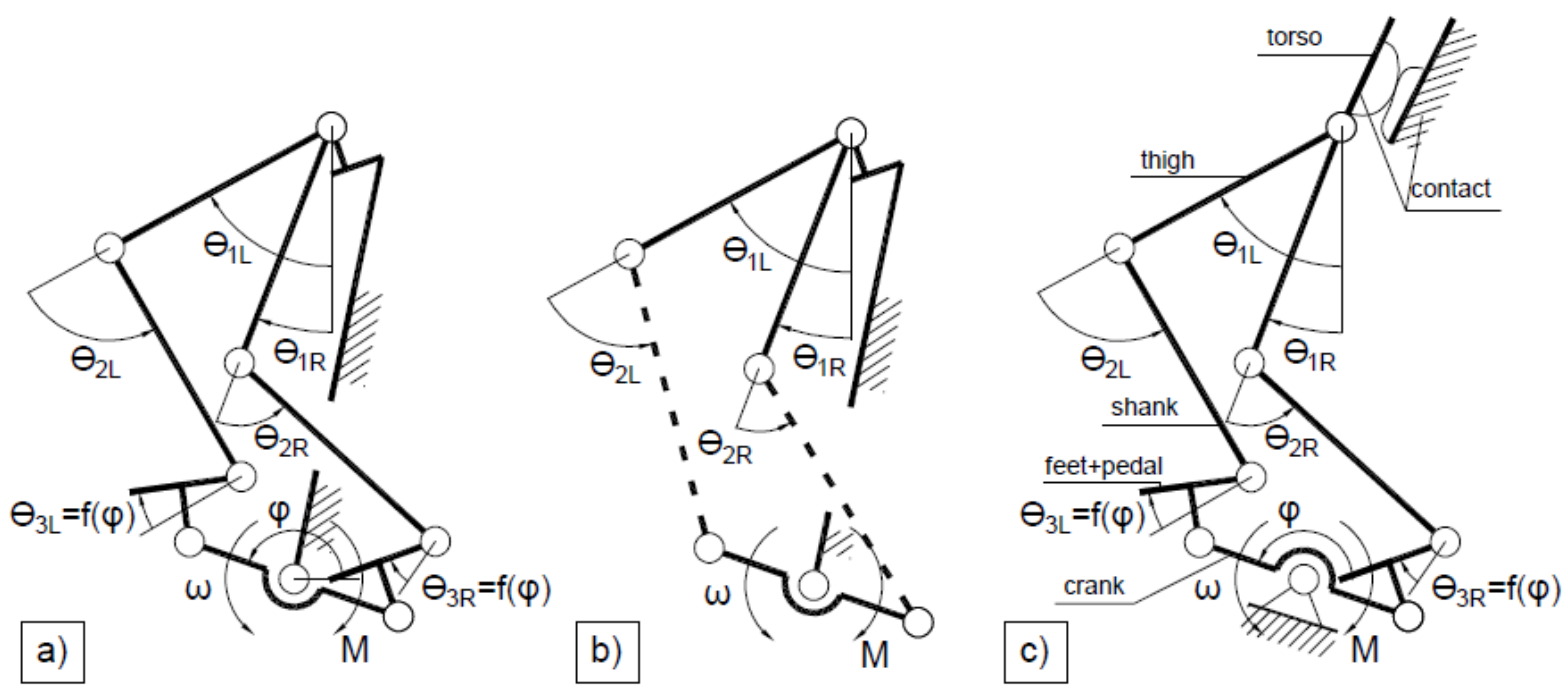

Fig. 1: Planar models of human-bicycle system: a) 2D3F0T, b) simplified 2D3F0T, c) $2 D 3 F 3 T$

\subsection{Elimination of passive constraints in the human-bicycle kinematic model}

Part of the work devoted to the inverse dynamics task assumes the coupling of movement in the ankle with the angle of the crank rotation (Bini, 2016). This is because the ankle joint in comparison to the knee and hip joint has the smallest range of movements in the sagittal plane - the plane of movement of the kinematic elements of the bicycle. There is also the possibility of omitting the ankle joint model in order to simplify the structure of a single lower limb to a form of a crank-rocker linkage (Fig. 1b). This model allows to determine the kinematic parameters of all parts of the limb and allows the active participation of only two joints in each limb.

To obtain reliable numerical data, the model should represent a system without passive constraints and not be a redundant system in relation to its number of drives. Therefore, when considering a direct kinematic task in which man drives the crank using actively all 6 joints in the sagittal plane, it is necessary to introduce additional 3 degrees of freedom into the model and 2 dependencies describing the angles in the ankles depending on the phase of the crank rotation (Fig. 1c).

Table 1 shows four models, two in a planar system and two in a spatial system. The 2D3L0T model was taken from the conducted inverse kinematics task studies (Takehara, 2012). The 2D3L3T model is its development and takes into account the lack of a kinematic pair between the torso and the bicycle saddle, only allows contact between them based on force, but does not prevent the torso from tearing off the saddle. The 3D6L3T and 3D7L5T models are based on a spatial system as well as additional degrees of freedom in the hip and ankle joints. Both spatial models take into account the moveable connection between the torso and the saddle. In the case of the 3D6L3T model, this association represents a kinematic pair allowing only three revolutions around the global coordinate system - the spherical joint. This model represents a simplification of the relationship between the upper limbs and the bicycle steering wheel. Simplification consists in maintaining a fixed point of contact between the body and the saddle, and takes into account the flexibility and the shape-force nature of the contact between the torso and the saddle. The last considered 3D7L5T model has an additional rotation about the geometrical axis of the shank. This rotation is in fact a redundant movement with respect to the required 6 degrees of freedom of the foot relative to the pelvis. To equate the number of active torques in this model with the number of degrees of freedom of the entire structure, two additional degrees of freedom between the torso and the saddle were allowed - a free displacements of contact points between the body and the saddle while maintaining a continuous contact - point to surface constraint. 
Tab. 1: Kinematics of studied models.

\begin{tabular}{ccccc}
\hline $\begin{array}{c}\text { Model } \\
\text { name }\end{array}$ & Model version & $\begin{array}{c}\text { Model } \\
\text { DOF }\end{array}$ & $\begin{array}{c}\text { Displacments (direct } \\
\text { kinematics) }\end{array}$ & $\begin{array}{c}\text { Active torques } \\
\text { (inverse kinematics) }\end{array}$ \\
\hline 2D3L0T & $\begin{array}{c}\text { 2D,3DOF legs, Torso } \\
\text { fixed }\end{array}$ & 3 & $\varphi, \Theta_{3(\mathrm{~L}, \mathrm{R})}$ & $\Theta_{1(\mathrm{~L}, \mathrm{R})}, \Theta_{2(\mathrm{~L}, \mathrm{R})}, \Theta_{3(\mathrm{~L}, \mathrm{R})}$ \\
\hline 2D3L3T & $\begin{array}{c}\text { 2D, 3DOF legs, 3DOF } \\
\text { Torso }\end{array}$ & 6 & $\varphi, \Theta_{3(\mathrm{~L}, \mathrm{R})}, \mathrm{T}_{\mathrm{x}}, \mathrm{T}_{\mathrm{y}}, \mathrm{R}_{\mathrm{z}}$ & $\Theta_{1(\mathrm{~L}, \mathrm{R})}, \Theta_{2(\mathrm{~L}, \mathrm{R})}, \Theta_{3(\mathrm{~L}, \mathrm{R})}$ \\
\hline 3D6L3T & $\begin{array}{c}\text { 3D,6DOF legs,3DOF } \\
\text { Torso }\end{array}$ & 6 & $\varphi, \Theta_{3(\mathrm{~L}, \mathrm{R})}, \mathrm{R}_{\mathrm{x}}, \mathrm{R}_{\mathrm{y}}, \mathrm{R}_{\mathrm{z}}$ & $\Theta_{1(\mathrm{~L}, \mathrm{R})}, \Theta_{2(\mathrm{~L}, \mathrm{R})}, \Theta_{3(\mathrm{~L}, \mathrm{R})}$ \\
\hline 3D7L5T & $\begin{array}{c}\text { 3D,7DOF legs, 5DOF } \\
\text { Torso }\end{array}$ & 10 & $\begin{array}{c}\varphi, \Theta_{3(\mathrm{~L}, \mathrm{R})},\left\{\alpha_{(\mathrm{L}, \mathrm{R})} \text { or } \beta_{(\mathrm{L}, \mathrm{R})}\right\}, \\
\mathrm{T}_{\mathrm{x}}, \mathrm{T}_{\mathrm{y}}, \mathrm{R}_{\mathrm{x}}, \mathrm{R}_{\mathrm{y}}, \mathrm{R}_{\mathrm{z}}\end{array}$ & $\begin{array}{c}\Theta_{1(\mathrm{~L}, \mathrm{R})}, \Theta_{2(\mathrm{~L}, \mathrm{R})}, \\
\Theta_{3(\mathrm{~L}, \mathrm{R})}, \alpha_{(\mathrm{L}, \mathrm{R})}, \beta_{(\mathrm{L}, \mathrm{R})}\end{array}$ \\
\hline
\end{tabular}

The 2D3L0T model obviously does not allow direct transition from inverse to direct kinematics task, while the 2D3L3T model allows such transition. However, due to the passive constraints present in such a system (assuming the limb structure in a spatial system), these solutions should be abandoned in favor of spatial models. A model having a torso with 3 degrees of freedom (Fig. 2a) can be used directly to move from the inverse to the direct task, in particular when using a numerical model.

In the case of the model with additional degrees of freedom of the torso (Fig. 2b) and the used structure of the lower limb with 7 degrees of freedom, there are more possibilities in determining the displacement characteristics in the given joints for the inverse kinematic task. The number of assumed movements should be consistent with the number of the parameters listed in the Table 1, and therefore, in addition to determining the 5 degrees of freedom of the torso, it is necessary to control the angles of rotation $\beta$ in the knee joints or angles $\alpha$ in the ankle joints.
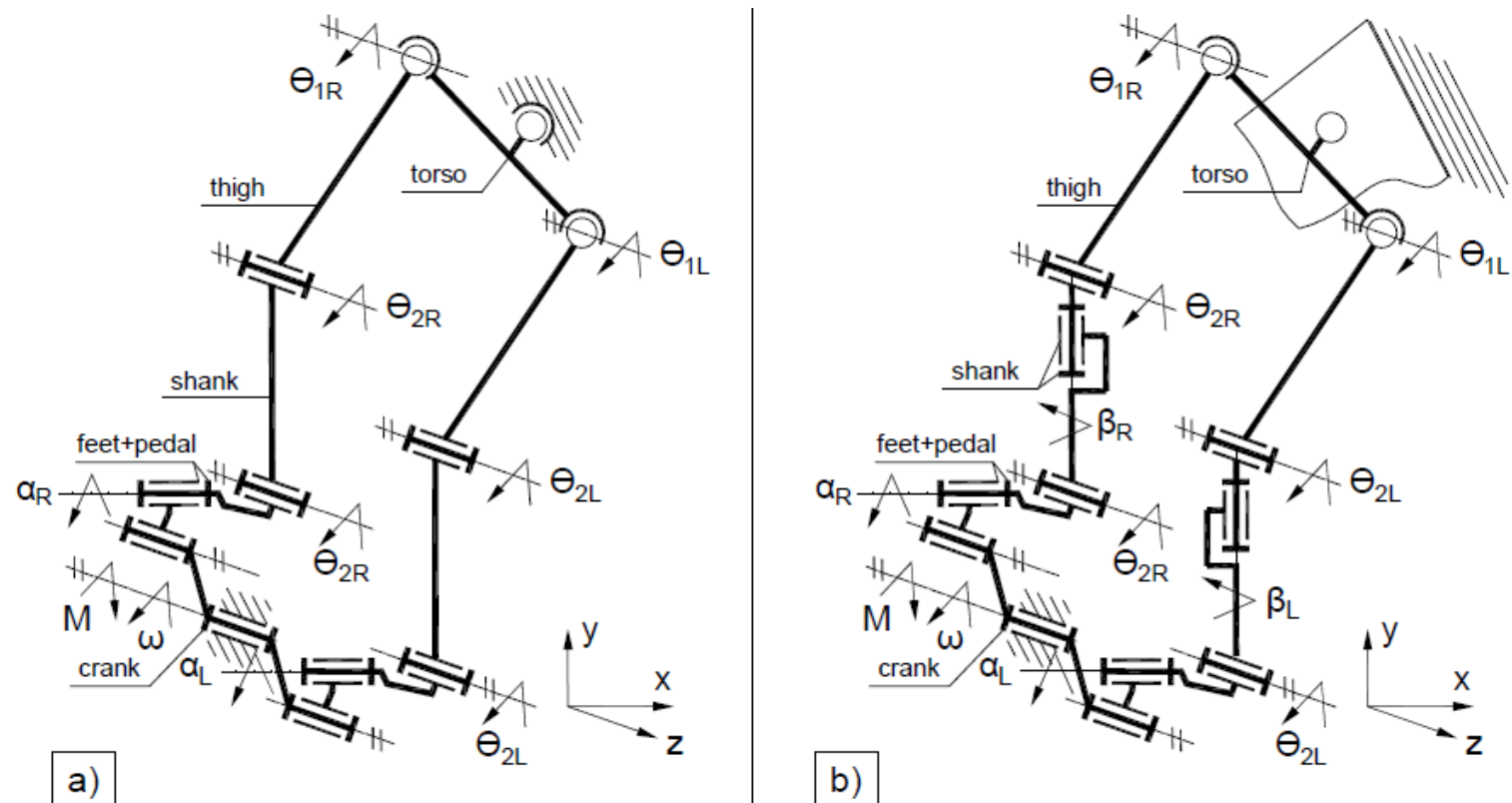

Fig. 2: Kinematic structure of spatial models: a) 3D6L3T, b) 3D7L5T.

\section{Preliminary results}

Based on the described kinematic diagrams (Fig. 2 a, b), a preliminary numerical model was created in the ADAMS environment using the appropriate dimensional data (Fig. 3a). On this basis, it was possible to carry out the inverse kinematics for an average cadence of $90 \mathrm{rpm}$. The results of the simulation were used to create displacement characteristics in all joints, where drives for the direct kinematics task were eventually introduced. The simulation tests were performed assuming full immobilization of the torso in 3D6L3T, 3D7L5T models and in the absence of $\beta$ displacements in the 3D7L5T model. The results obtained using direct kinematics task are presented in Fig. $3 \mathrm{~b}$ in the form of active moments for the hip joints of both considered models in the sagittal plane. 
a)

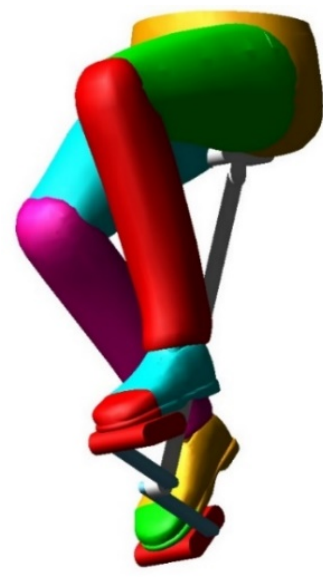

b)

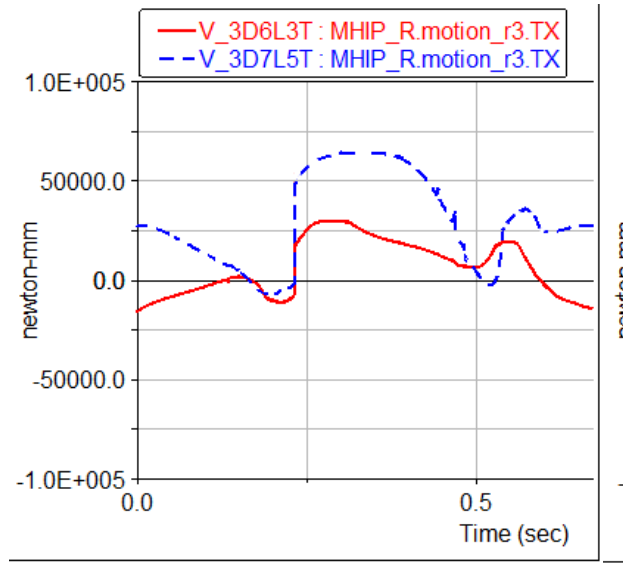

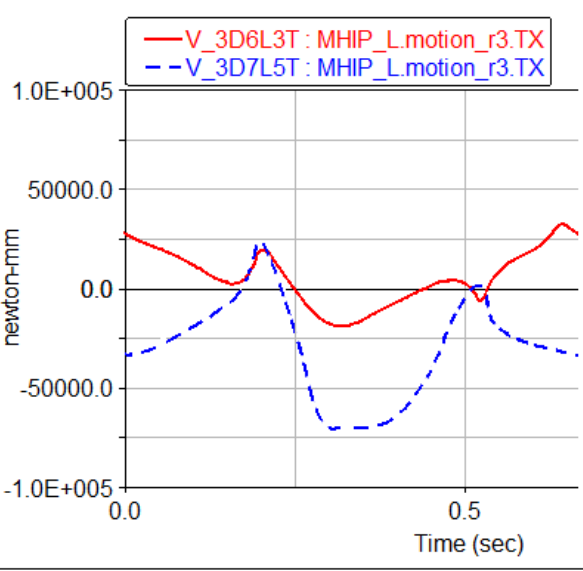

Fig. 3: a) numerical model created in ADAMS, b) Preliminary results of hip joint torques for 3D6L3T and 3D7L5T models

\section{Conclusions}

Based on the conducted research, it can be concluded that the majority of displacements in individual joints while turning the crank of the bicycle indicate that the movements are planar due to the planar kinematic system of the bicycle itself. The results of the analyzes carried out, however, confirm the necessity of building spatial models describing the relationship between man and bicycle. The main reason is the spatial structure of the lower limb itself. In addition, maintaining the central points of the hip, knee and ankle joint in the normal plane to the axis of the crank requires additional energy consumption. The problem is the adoption of a specific relationship between the trunk and the saddle. Man in fact creates a rigid connection with the mechanical system of the bicycle only by grasping the steering wheel and attaching the feet to the pedals. Therefore, the choice of a particular structure of the man-bike model determines its research capabilities in terms of the impact of the driving style on selected dynamic parameters.

The presented work is an introduction to further and wider research, in which a well-developed biomechanical man-bike model will be needed. The main goal of further work will be to extend the model being developed to include upper segments of the human body. Further research will include, among others analysis of the ability to treat posture and joint defects (deformity of the knees) on the basis of introduced modifications in the construction of mechanical components of the bicycle.

\section{References}

Arkesteijn, M., Jobson, S. A., Hopker, J. G. and Passfield, L. (2016) The effect of cycling intensity on cycling economy during seated and standing cycling. International journal of sports physiology and performance, 11 (7). pp. $907-912$

Bini, R. R., Dagnese, F., Rocha, R., Silveira, M. C., Carpes, F. P. and Mota, V. B. (2016) Three-dimensional kinematics of competitive and recreational cyclists across different workloads during cycling. European Journal of Sport Science, Vol. 16, Issue 5, pp. 553-559

Olinski, M., Gronowicz, A., Handke, A. and Ceccarelli, M. (2016) Design and characterization of a novel knee articulation mechanism. International Journal of Applied Mechanics and Engineering, Vol. 21, no. 3, pp. 611622.

Puchera, J., Buehler, R. and Seinena, M. (2011) Bicycling renaissance in North America? An update and reappraisal of cycling trends and policies. Transportation Research Part A: Policy and Practice. vol. 45, Issue 6, pp. 451-475

Takehara, S., Murakami, M. and Hase, K. (2012) Biomechanical Evaluation of an Electric Power-Assisted Bicycle by a Musculoskeletal Model. Journal of System Design and Dynamics, Vol. 6, No. 3, pp. 343-350 